This is the peer reviewed version of the following article: [Bourret, P., and Cambrosio, A. (2019)1 Genomic expertise in action: molecular tumour boards and decision-making in precision oncology. Sociology of Health \& IIIness 41, 1568-1584.]

Genomic expertise in action: molecular tumour boards and decision-making in precision oncology

Pascale Bourret ${ }^{\mathrm{a}}$, Alberto Cambrosio ${ }^{\mathrm{b}}$

${ }^{\text {a }}$ Aix-Marseille Univ, INSERM, IRD, SESSTIM, Institut Paoli-Calmettes, 232 Bd SainteMarguerite, 13273 Marseille CEDEX 9, France. Email: pascale.bourret@univ-amu.fr ${ }^{b}$ Department of Social Studies of Medicine, McGill University, 3647 Peel St., Montreal, QC, Canada H3A 1X1. Email: alberto.cambrosio@mcgill.ca

Corresponding author: Pascale Bourret, UMR 1252 SESSTIM, Institut Paoli-Calmettes, 232 Boulevard Ste Marguerite, 13273 Marseille Cedex 9, France. E-Mail: pascale.bourret@univamu.fr 


\title{
Genomic expertise in action: molecular tumour boards and decision-making in precision oncology
}

\begin{abstract}
The recent development of cancer precision medicine is associated with the emergence of 'Molecular Tumour Boards' (MTBs). Attended by a heterogenous set of practitioners, MTBs link genomic platforms to clinical practices by establishing 'actionable' connections between drugs and molecular alterations. Their activities rely on a number of evidential resources e.g. databases, clinical trial results, basic knowledge about mutations and pathways - that need to be associated with the clinical trajectory of individual patients. Experts from various domains are required to master and align diverse kinds of information. However, rather than examining MTBs as an institution interfacing different kinds of expertise embedded in individual experts, we argue that expertise is the emergent outcome of MTBs, which can be conceptualized as networks or 'agencements' of humans and devices. Based on the ethnographic analysis of the activities of four clinical trial MTBs (three in France and an international one), and of two French routine-care MTBs, the paper analyses how MTBs produce therapeutic decisions, centring on the new kind of expertise they engender. The development and activities of MTBs signal a profound transformation of the evidentiary basis and processes upon which biomedical expertise and decision-making in oncology are predicated, and, in particular, the emergence of a clinic of variants.
\end{abstract}

Keywords. Molecular Tumour Boards; Precision Medicine; Cancer Genomics; Bio-Clinical Expertise

\section{MTBs, a novel institution for precision oncology}

The therapeutic management of cancer patients is generally based on standardized treatment plans which are in turn embedded in clinical guidelines. Today's oncology is a highly protocolized activity: new, experimental protocols are tested in clinical trials and rapidly turned into routine ones. Clinical oncologists only enter a phase of 'tinkering' after all standard therapeutic interventions have failed (Baszanger 2012). When confronted with complex situations, such as those characterized by the absence of validated standards or for which significant changes in therapeutic strategy might be considered, oncologists can resort to 'tumour boards' (TBs). In France, this has become mandatory since 2003. TBs bring together clinicians from different specialties (oncologists, pathologists, surgeons, radiotherapists), often specializing on a particular, organ-defined form of cancer, who discuss 
difficult cases to reach a collective, consensus-based therapeutic decision (Kuroki et al. 2010, Castel et al. 2012). In recent years, the development of 'precision oncology' has led to the emergence of a new kind of TBs, generally referred to as 'Molecular Tumour Boards' (MTBs). Attended not only by the aforementioned clinicians but also by scientists (namely molecular biologists, geneticists, pathway specialists, biostatisticians, computational biologists, and bioinformaticians), and reaching across different types of cancer, MTBs provide a bio-clinical platform to assess the clinical significance of the results of genomic tumour profiling and make therapeutic recommendations on that basis.

Oncology practitioners themselves have called attention to the development of MTBs as a noteworthy characteristic of the emerging field of precision oncology (Schwaederle et al. 2014, Tafe et al. 2015, Erdmann 2015, Knepper et al. 2017, McGraw et al. 2017, Stoeklé et al. 2017, Horak et al. 2017, Hoefflin et al. 2018). The purpose of these practitioners' contributions is to document and promote the role and practices of these novel institutions, whose multidisciplinary line-up is justified by relation to the introduction of innovative diagnostic and therapeutic approaches such as targeted therapies, immunotherapies, molecular biomarkers, and next-generation sequencing techniques (NGS). These innovations, we are told, mobilize the expertise of a number of different practitioners whose skills and contributions are necessary to master and align the disparate kinds of information upon which the interpretation and evaluation of the results of high-throughput genomic testing are predicated. Initially mostly confined to clinical trials, MTBs have recently made inroads into routine care settings increasingly characterized by an experimental orientation (Cambrosio et al. 2018). While MTBs can be said to share a common purpose with TBs, namely the enactment of consensual therapeutic decisions for individual patients, the work required to accomplish this task is different. In the case of MTBs, it consists in interpreting the results of tumour genomic profiling in order to identify and select the molecular alterations to be targeted by specific drugs; in other words, to identify and prioritize actionable alterations.

The notion of actionability (Nelson et al. 2013) is key in this respect: it distinguishes somatic precision oncology from other domains, such as medical genetics. While the latter has also embraced high-throughput genomic techniques and established molecular boards to interpret results, its focus is on the diagnosis and management of inherited risks (Timmermans et al. 2017). These diagnostic activities have led to the emergence of a 'clinic of mutations' aimed at documenting "the biological nature and the clinical relevance of mutations, whose status is ambiguous and whose effects are uncertain" (Rabeharisoa and Bourret 2009: 691). Actionability regimes, in contrast, aim to predict relationships between genomic information and drug therapies, thereby extending into new clinical territories. 
Rather than simply broadening the activities of TBs by incorporating additional laboratory results, MTBs perform a different kind of work. In a domain characterized by rapidly evolving, often "fuzzy" (McGraw et al. 2017) knowledge, their task is to determine the presence of genomic alterations known as variants in patients' tumours, to assess the evidence supporting their possible role in disease development, and, in the case of multiple alterations, prioritize targets for therapeutic intervention. Once combined with clinical information about individual patients (e.g. their response to previous treatments), these evaluations will lead to specific therapeutic recommendations. As we will see, in the case of MTBs actionability is thus both the raison d'être of these new institutions and the outcome of their deliberations. We propose to call this new kind of activity a clinic of variants, for the following reasons: because of its clinical nature that distinguishes it from pre-clinical work, its direct therapeutic purpose that differentiates it from more traditional diagnostic work, and its unprecedented granular focus on variants rather than genes. As embodied in MTBs, the clinic of variants rests on a heterogeneous assemblage of people and devices that, acting together, lead to clinical decisions. But how, exactly, can we account for these activities?

\section{Investigating MTBs: conceptual musings}

As mentioned in the previous section, MTB activities, while defined by their common goal of enacting actionable interpretations, are characterized by the presence of a number of different specialists, namely clinicians (medical oncologists, surgeons, radiologists and radiotherapists, pathologists), biologists (geneticists, molecular biologists), biostatisticians, and information technology specialists (computational biologists, bioinformaticians). Each of these practitioners can be said to possess peculiar skills, i.e. distinctive forms of expertise that require the mastery of specific tools, a shared language, and shared assessment criteria. It is thus tempting to analyse MTBs as trading zones, i.e. to equate them to situations where actors operating under different conceptual schemes or paradigms need nonetheless to communicate for the purpose of exchanging objects or collaborating (Galison 1997, chapter 9). Trading zone participants develop a contact language that allows for situated interactions and the local interfacing of objects and material entities even when they disagree on the meaning of the objects being exchanged or the goals of the collaboration. For a number of reasons, however, resort to the notion of trading zones in the case of MTBs turns out to be problematic.

Consider gene variants identified by NGS: the issue is less one of interfacing multiple preexisting interpretations than to produce a de novo (clinical) interpretation of those variants by collectively assembling a number of elements, ranging from clinical to biological and 
computational considerations, as related to a specific clinical case. This kind of interpretation is strategically oriented towards clinical decision-making, i.e. the definition of a therapeutic intervention. The actionability of a given variant, in other words, rather than a stable property of that variant is the emergent outcome of recursive processes. Irreducible to linguistic exchanges, MTB activities amount to a collective, decision-oriented practice that displays the following characteristics: (a) a sui generis nature (as Durkheim would have it), insofar as MTB recommendations cannot be reduced to individual judgments or to the mechanical application of rules; (b) a heterogeneous configuration, given that MTB recommendations rely not merely on the mobilization of human skills, but also on the deployment of distinctive tools and equipment (e.g., data repositories) that sometimes provide conflicting definitions of variants; and (c) a situated, ad hoc dimension that oversees the transition from the potential, in principle actionability of particular alterations to actual, in practice recommendations for specific patients.

Our criticism of the notion of trading zones also applies, mutatis mutandis, to contributions that have analysed MTBs in terms of a "pooling [of] professional expertise" (McGowan et al. 2016: 6). By merely focusing on the presence of different experts (albeit acknowledging the fact that new, non-clinical specialists have joined the fray), and even when emphasizing "transdisciplinary engagement" rather than mere multi-disciplinarily (Knepper et al. 2017: 146), this kind of account fails to capture the specificity of MTBs as compared to more traditional TBs. We suggest that only a focus on the actual content of MTB activities can lead to an understanding of the peculiar kind of expertise they deploy, and that in order to pursue this line of inquiry we need to examine MTBs, rather than its individual members, as the source of the bio-clinical expertise that underlies the clinic of variants. In other words, our focus is on expertise as a process and a network rather than a property of individuals (Eyal 2013, Cambrosio et al. 1992). As such, we can empirically investigate the conditions of possibility for this distributed form of expertise, the resources it requires, as well as its contingent and temporal dimensions. Rather than a juxtaposition of experts defending their respective jurisdictions, MTBs are characterized by 'expertise in action' as evidenced by exchanges whereby clinicians engage in biological and pharmacogenomic arguments on molecular pathways and the activity of different drugs, while molecular biologists do not refrain from discussing the clinical aspects of variant analysis. MTBs, from this perspective, qualify as assemblages (or better: agencements, a term that denotes both the process and the result) that in addition of being composed of heterogeneous elements, display immanent, emergent properties but also downward causality, for once in place they act on their components (DeLanda 2016). 


\section{Materials and methods}

Between 2015 and 2018, we accessed a total of 96 clinical trial MTB teleconferences and meetings. Two trials, a flagship French clinical trial (73 MTBs) and an international (EuropeNorth America) trial (17 MTBs), were our main source of data. We also attended 16 steering committee meetings of the same French trial and were given access to archival material concerning a second one. In order to obtain additional background information, we attended three MTBs from each of two other French clinical trials. In most cases, we were able to audiotape and transcribe MTB discussions; in the remaining cases we took detailed fieldnotes. Except for the international trial where discussions took place in English, meetings were conducted in French. All translations are ours. Teleconferences were of variable duration, ranging from as little as $\mathbf{2 0}$ minutes to one-hour meetings, depending on the number of patients discussed, the number and degree of involvement of attending members, and the complexity of genomic results and clinical trajectories discussed during each MTB. As explained in the next section, attendance varied widely, ranging from as few as 5 to 25 participants.

To complement the information obtained from fieldwork observations, we collected documents related to 215 MTB meetings from our main French trial, including genomic results, minutes of therapeutic decisions, and e-mail exchanges between MTB members debating contentious or uncertain cases in the aftermath of an MTB meeting. We were also able to consult genomic reports for the MTBs of the international trial. We interviewed 40 MTB participants and related practitioners from all the trials, including trial PIs, molecular biologists, clinical oncologists, biostatisticians, bioinformaticians, and trial coordinators. In addition to the aforementioned material related to clinical trial MTBs, we attended 25 MTBs in routine clinical settings in two French cancer centres. These MTBs, which discussed advanced or rare cancer patients, corresponded to the project of 'transitioning' this new kind of institution from a clinical research to a care setting, thus acting "as a translational device for integrating research and care" (Cambrosio et al. 2018: 214).

Teleconference transcripts, interviews, fieldnotes, and relevant publications were compiled into memos that were circulated amongst the two co-authors. In order to develop an empirically grounded framework for analysing MTB activities, we coded these materials for recurring themes using qualitative data analysis methods (e.g., Bourgeault et al. 2013). In particular, we processed the information we collected "against a background of cultivated theoretical expertise" (Timmermans and Tavory 2012: 180). Given the often highly technical nature of the topic, and with our readers in mind, the excerpts from MTB discussions used in this paper combine simplified summaries of the context of the debate with verbatim extracts 
(in italic). All fieldnote excerpts and quotations used in this paper come from the French clinical trial that was our major source of data. Similar evidence is available from the other trials, in particular the international one, but we refrained from quoting these data because of a number of issues (differences in protocol, national regulations, and access to therapies) that would have required further complex explanations, thus unduly lengthening the paper without affecting its key arguments.

The results reported in this paper are part of a broader project on translational research, actionability, and clinical utility in precision oncology. The project has been approved by the Institutional Review Board of the McGill Faculty of Medicine (IRB Study Number A02-E1914B). Written consent for observations was obtained from all sites and written or oral consent for interviews from individual informants.

\section{MTBs in action}

MTBs are flexible institutions: when associated with clinical trials, their meetings are in principle scheduled at fixed periods (weekly or biweekly) but they can be postponed depending on patient enrolment and the availability of genomic results. Attendance also varies from meeting to meeting. In the case of multisite clinical trials, practitioners from several centres can in principle attend the teleconferences, and they often (but not always) do when discussions concern their patients. The main French trial we followed mobilized close to 30 recruiting sites, which meant that about 50 practitioners could have in principle attended the MTB, although, in practice, attendance varied between 10 to 20 participants. In addition to the trial coordinator who acts as the 'guardian of the protocol', reminding participants of the rules they should follow (but can also alter if deemed necessary: see below), the 'core set' of attending practitioners consisted of the clinical trial PIs and the molecular biologists from the technical platforms performing the genomic analyses.

MTB meetings always begin with a clinical case report, i.e., a narrative summarizing a patient's clinical diagnosis, disease trajectory, and previous treatments. Compared to more traditional staff meetings or hospital teaching-ward rounds, the case report is in general quite short. Participants, however, when faced with either ambiguous genomic results or different therapeutic options, can subsequently ask for precisions about the patient's previous clinical trajectory to make better informed decisions. Case presentation is quickly followed by a summary of the results of genomic analyses, listing the variants that qualify as possible tumour drivers and could thus be targeted by available drugs. In the case of three trials, a separate molecular-biology team meeting had initially been scheduled to assess 
genomic results. This arrangement, however, was quickly abandoned when it became clear that joint discussions between clinicians and biologists were necessary to make full sense of the results. Some clinical trials (one in our sample) use the services provided by commercial companies such as Foundation Medicine, whose reports follow a standard format and include suggestions of possible treatments with supporting evidence. These results and related suggestions, however, are not necessarily validated during MTB discussion. Results produced by local laboratories follow more open-ended formats and often include visual diagrams that participants can inspect to avoid the black-boxing of genomic analyses. Presentation of the genomic results is followed by a general discussion that centres on the actionability of the genomic variants and their prioritization in the case of multiple variants. The MTB ends with a decision about a recommended drug-variant match.

By analogy with Garfinkel's (1984) study of jury members, we are interested in understanding how MTB members produce themselves within an MTB meeting as an MTB. In other words, we are not interested in describing or compiling the sociodemographic or professional characteristics of individual MTB members, but in understanding MTBs as sui generis institutions for performing precision oncology. This involves investigating the content of their activities by focusing not solely on human participants, but also and simultaneously on the 'equipment' that is constitutive of MTB decision-making. We will thus approach MTBs as peculiar heterogeneous agencements, and investigate the methods, including the equipment, they deploy to reach therapeutic decisions, as well as the temporality and situatedness of their activities.

\subsection{MTBs as collective bio-clinical expertise}

Our fieldwork provided rich material to analyse the peculiar kind of expertise deployed by MTBs. As discussed in Section 2, some observers have analysed MTB activities in terms of the pooling of different sorts of expertise, arguing that although new actors, e.g. biologists, had to be included because their expertise was required for the interpretation of genomic analyses, therapeutic decision-making rested firmly in the hands of clinicians. Otherwise put, while taking into account the opinions of their scientific colleagues, clinicians had the final word concerning the interpretation of the clinical significance of genomic variants. This account does not correspond to the modus operandi of the MTB meetings we observed. One cannot dissociate the interpretation of genomic variants from therapeutic decision-making based on that interpretation. Under the regime of actionability that characterizes precision oncology, the interpretation of a given variant includes the potential effects of specific drugs on that variant. In other words, genomic variants, within such a framework, are not merely 
diagnostic 'biomarkers', they are simultaneously drug targets, i.e. they are defined in relation to specific therapeutic agents. In return, they provide information on the functional role of those variants. (Vignola-Gagné et al. 2017).

The following excerpt exemplifies our claim that MTBs deploy a new kind of expertise that is neither clinical nor biological, but the emergent result of collective bio-clinical activities. As such, it cannot be reduced to the pooling of a number of pre-existing professional skills:

The genomic analysis of a lung cancer patient finds a mutation in the BRAF gene: not the most common V600E variant, against which the [much-touted] targeted drug Vemurafenib has been developed and marketed, but the D594N variant that, according to trial protocol, can be targeted with experimental Drug Y. A molecular biologist (MB1) mentions that according to another, ongoing clinical study, D594N doesn't qualify for treatment with Vemurafenib. He doesn't know, though, whether this also applies to Drug $Y$, and wonders whether "clinicians might want to give it a try knowing that the drug in our trial is Drug $Y$, not Vemurafenib". The trial administrative coordinator (TAC) asks whether there is any evidence concerning Drug Y: How did practitioners establish that Vemurafenib doesn't work with D594N? How does this apply to Drug Y? MB1 replies that he doesn't know the answer to these questions. The oncologist PI (TPI) shifts the debate by stating that in fact Drug $\mathrm{Y}$ is not directed against BRAF, but against another cancer gene (MEK) located downstream in the same molecular pathway. He argues that "taking into account that D594N is an activating mutation, that the patient is a light smoker without any of the other mutations usually associated with lung cancer, it doesn't seem illogical to try Drug Y". Another oncologist supports the TPI argument. TAC summarizes the discussion but stating that "we therefore consider that D594N is an activating mutation and that Drug $Y$ will act on the downstream pathway". The written summary of the decision reads as follows: "The BRAF D594N variant is likely to be of no diagnostic or treatment values. ... In [a concurrent study], D594N is not eligible for treatment with Vemurafenib because it confers resistance to this drug. However, because Drug $Y$ targets the pathway downstream of BRAF, D594N will make the patient eligible for this treatment".

In this excerpt, because no other actionable alterations had been detected, the patient was prescribed an experimental drug based on hypotheses about its potential activity on genes and variants in the downstream pathway. To reach this decision, clinicians and biologists combined their knowledge about molecular pathways and drug action. While individual actors might have had access to specific elements not readily available to others, no a priori professional hierarchy between MTB participants was apparent. Indeed, both clinicians and biologists contributed and triangulated different yet overlapping bits of information about possible drug behaviour, without any jurisdictional delimitation of domains of expertise. The oncologists' arguments show that they integrated molecular-biological elements into their clinical reasoning and suggestions. This kind of sui generis discussion allowed the MTB to 
reach a therapeutic decision for this particular patient and to simultaneously generate hypotheses about the mechanisms of action and the use of targeted therapies. This dual achievement was not predicated upon a mere interfacing of different areas of individual expertise. Rather, it was the immanent result of this peculiar MTB assemblage.

The following excerpt provides another example of how MTB activities correspond to the emergence of a hybrid and collective form of expertise that cuts across cognitive and jurisdictional boundaries between pre-existing domains.

According to protocol, a breast cancer patient needs to undergo a second line of traditional chemotherapy before receiving a targeted therapy as part of the clinical trial. The tumour has evolved since her initial diagnosis and now belongs to the aggressive category known as 'triple-negative' (because it lacks the three main receptors). Genomic results show the presence of an AKT1 mutation that is strongly indicative of a different kind of breast cancer, called 'luminal', that corresponds to the initial diagnosis. The discussion centres on whether the second line therapy should be based on the luminal or the triple-negative diagnosis. The treating oncologist recommends a standard chemotherapy drug (Eribulin), which is generally used in the case of aggressive tumours.

This suggestion leads to an animated discussion: the oncologist PI [TPI] hesitatingly states: "I don't know ... because the genomic profile ... the molecular biologist [MB] is in a better position to comment the situation, it looks more luminal than triple-negative". MB confirms that the mutation "is strongly indicative of luminal". A pathologist argues that "phenotypically [as determined by pathology] she is triple-negative". The TPI speculates that "maybe she lost the hormone receptors, but the tumour still behaves like a luminal one". Another oncologist notes that "even so, her tumour is resistant to Taxol [a major chemotherapy drug], it's a very aggressive kind of cancer". The TPI summarizes the resulting consensus as follows: "We all agree that she has an AKT1 mutation ... we can give her Eribulin for her second therapy line ... and then prescribe an anti-AKT1 drug as part of the targeted-therapy trial".

In this case the MTB could not rely on previous examples of how to deal with a situation characterized by a lack of alignment between genomic and clinical parameters. The chosen solution, based on a mixture of clinical, genomic, and pharmacological considerations, articulated a traditional chemotherapy treatment according to the patient's clinicalpathological status with a subsequent targeted-therapy treatment according to the genomic profile. A quite unusual therapeutic decision, it linked two different diagnoses of the same tumour as part of a sequential strategy. Within the context of the MTB, the performance of a core component of clinical work, namely diagnosis, deviated from the traditional understanding of the clinician's autonomous decision-making, and mobilized instead a mixture of clinical and molecular biology reasoning jointly produced by MTB participants. 
As shown in this section, MTB decisions cannot be reduced to independent pronouncements by individual MTB members, but are the result of a collective dynamics that transgresses disciplinary and specialty boundaries. MTB activities, however, are also irreducible to verbal exchanges between human actors. To fully investigate how an MTB, as a whole, 'thinks', and to account for the kind of collective expertise it engenders we need to include in our analysis the equipment that acts as a condition of possibility for its activities. In other words, while it could be argued that only humans make decisions, an exclusive focus on humans will miss the heterogenous sources of MTB collective decision-making, a task that is contingent on real-time access to a number of tools and objects, as discussed in the next section.

\section{2 Equipping and engendering expertise: expertise as a network}

The performance of MTB activities requires the mobilization of a number of devices. In addition to reports and visual inscriptions derived from the high-throughput analysis of patient material, they include generally available databases and, more specifically, 'knowledgebases' that provide access to highly curated functional information about variants (Cambrosio et al. forthcoming), various kinds of software, but also convention-based tools such as Levels of Evidence tables (e.g. Mateo et al. 2018) designed to obviate the lack of standards by helping MTB members assess the trustworthiness of drug-biomarker matches. MTBs will also implement local tools such as algorithmic decision trees that, while open to revisions, are included in trial protocols. In other words, in order to carry out its work, MTBs need to be properly equipped, although we should quickly add that equipment itself becomes a topic of investigation when it provides uncertain or conflicting information, as it often happens. To claim that MTB expertise is a property of a (temporarily stable) network amounts to arguing that, while knowledge and know-how can be said to be embodied in persons, they "cannot function as 'expertise' unless they become part of a network without which these skills could not be expressed or activated" (Cambrosio et al. 1992: 345). The following excerpt exemplifies this dynamic:

Biologist (BIO) 1 explains that the NGS results [of a breast cancer patient] show a TSC2 missense mutation that "is non-pathogenic according to SHIFT [a functional prediction software] and there is no associated $\mathrm{LOH}$ [loss of the second allele], ... plus it's a missense on a suppressor gene". He adds that "there is a mutation on NOTCH4, which can be found in databases of SNP polymorphisms, but 2\% only, which is bizarre because if germline it should be 25\%, and in the CGH [Comparative Genomic Hybridization] analysis NOTCH4 is normal, so this NOTCH4 mutation is kind of ambiguous". Concerning KDR [another gene], "there is indeed a mutation around $10 \%$, but it needs to be double-checked because it's out of the kinase domain, and here again, it's described as non-pathogenic in the PolyPhen [database]... so this 
is not very theragnostic". To sum up, "with NGS we have nothing of a real theragnostic interest". BIO2 then describes additional results of the CGH analysis, stating that it "found a clear amplification of FGFR1".

The MTB discusses which alteration to choose given that according to rules, mutations have priority over amplifications. The MTB decides, however, to opt for the amplification on the basis of information provided by databases and prediction algorithms for that specific kind of TSC2 mutations. $\mathrm{BIO} 1$ sums up the situation: "TSC2, there is no loss of the second allele, and it's a suppressor gene, it's not a truncating mutation, it's a missense mutation and the algorithms suggest that the missense mutation is not pathogenic, so...". BIO2 concurs, arguing that "above all, with CGH we have a FGFR1 amplification, 1.7 megabases, and log-wise above 3 , we could retain that one". The following short exchange ensues:

Oncologist (ONCO) 1: OK, so obviously this one instead of TSC2.

ONCO2: We thus opt for Drug $X$ [that targets the amplification].

ONCO1: It's clear-cut and logical.

This excerpt clearly illustrates the role of databases and knowledgebases as socio-technical prostheses that allow the MTB to reach temporary closure without fully determining it. The collective, sequential form of reasoning displayed by MTB members (including biologists who, far from merely providing technical information, are full-fledged reasoning partners) rests decisively on the triangulation of results provided by different kinds of equipment (NGS, $\mathrm{CGH}$ ) with the information about pathogenic variants derived from dedicated knowledgebases or, is some cases, elicited from ad hoc searches in publication databases such as PubMed. The previous excerpt, in other words, shows both the necessary role played by generally available data repositories (in most cases freely accessible), and the fact that the information provided by these repositories needs to be aligned with the bio-clinical results derived from individual patient tumours. Also notice how a somewhat meandering sequence of arguments (even in our simplified summary) transmogrifies in the end into a "clear-cut and logical" conclusion. This retrospective reconstruction of the line of reasoning that led to the MTB decision is not to be understood as a misappreciation by MTB members of the actual functioning of their debates but as akin to the "accountably 'adequate' rendering" of patient histories that takes place in ordinary medical encounters (Berg 1996: 519), albeit within the radically different context of a clinic of variants.

The peculiar kind of equipment mobilized by MTBs is a resource for decision-making, but also a topic of investigation and debate within MTBs, for instance when knowledgebases offer limited or contradictory information, or information that MTB members consider irrelevant or too generic for the specific case under discussion. These 'dispositifs' (Dodier and Barbot 2016; for the canonical definition see Foucault 1994: 299) act nonetheless as a condition of possibility for the production of a form of expertise that is defined by the open-ended 
agencement of human and non-human resources, often reframing discussions. Data repositories, moreover, anchor the expertise emerging from MTBs in the existence of broader networks of genomic practitioners who produce and update the tools and devices mobilized by MTBs, often in connection with their local MTB. The rapid evolution of the field (sometimes incremental, but occasionally disruptive, as with the emergence of immunooncology), accounts for the fact that knowledgebases and other similar devices cannot rely on the existence of stable, standardized forms of knowledge and practices, and thus contain uncertain and sometimes contradictory information. In the absence of standards, conventions can act as (temporary) substitutes.

Indeed, the excerpt also points to the existence of a number of conventions impinging on the deliberations of individual MTBs. The term "convention" refers, in this context, to agreements based on facts produced and tested collectively, regardless of whether these facts are entirely conclusive (Rabeharisoa and Bourret 2009: 712n32). These conventions can be part of a trial protocol but also be the object of ad hoc stipulations that can be more easily modified if needed. A set of 'rules' will for instance not only list the specific alterations that should be taken into account, but also specify the criteria defining those alterations. Thus, the 'decision guide' for one of the trials stated that amplifications should be considered as potentially actionable only if they fell within a threshold defined by a number of technical parameters (size, fold-change, LogRatio). This conventional threshold allowed practitioners to distinguish between, on the one hand, "real focal amplifications" affecting a small region of the chromosome and a small number of genes, and, on the other hand, large chromosomal gains that rendered the targeting of specific genes extremely uncertain. 'Rules' also often concern situations characterized by the presence of several alterations that need to be prioritized, for instance by giving precedence to mutations over amplicons. These prioritization rules, while referring to scientific evidence consigned in publications and knowledgebases, are also strongly derivative of the objectives of the trial, the drugs and panel of genes that are available as part of a specific trial, and other analytical parameters. They thus vary from trial to trial, although various organizations such as the European Society of Clinical oncology have launched initiatives to harmonize, for instance, the criteria defining Levels of Evidence for assessing the actionability of genomic alterations (Mateo et al. 2018). Efforts are also underway, for instance by the Variant Interpretation Cancer Consortium to harmonize the content of knowledge bases (Wagner et al. 2018).

The following excerpt illustrates a further aspect of the malleability of rules:

A clinical trial patient has a "borderline" molecular amplification, i.e. just above the threshold that would qualify it as actionable. If deemed actionable the alteration will make the patient 
eligible to receive Drug X; if not, no treatment will be available for this patient. According to the oncology PI, the decision rests on how the other MTB participants define the trial: " $a$ learning endeavour to produce new knowledge, or a trial to test and validate a given strategy". If the latter, one should stick to a strict definition and consider the molecular alteration as not-actionable. But "while this is a randomized trial [i.e., one designed to validate a strategy], it also qualifies as a trial with a learning component about how to define an oncogene from a bioinformatics and biological point of view, and this patient can help us to produce this kind of information".

In the excerpt, the oncologist is proposing an ad hoc revision of the rule-defined criteria in order to provide the patient with a treatment that would otherwise be unavailable to her. He does so by evidencing the indexicality of rules and rule-making, i.e. their connection with the purpose of the trial: without renouncing to the trial's testing dimension that requires strict adherence to rules, he also summons up its experimental nature (a "trial for learning") to justify a degree of flexibility in the application of rules, thereby also linking the experimental component of the clinical trial to its caring dimension.

In addition to ad hoc adjustments, MTB deliberations concerning specific patients can lead to more stable modifications that require official protocol amendments, as shown by the following excerpt.

A lung cancer patient has a KDR mutation that can be targeted by two available drugs ( $X$ and Y). According to trial protocol, however, these drugs should only be used for KDR amplifications, not KDR mutations. A molecular biologist (MB1) questions this rule, stating that the list of drugs and targets contains "bizarre" elements. In the ensuing debate the participants look for ways of justifying the prescription of Drug $Y$ to the patient based on the KDR mutation. The trial administrative coordinator (TAC) states that this goes against the rules, but MB1 counters this argument by pointing to past discussions about the inclusion of the KDR mutation amongst the targetable entities. The oncologist PI (TPI) states that he does not object to prescribing drug $\mathrm{Y}$, even if it means altering the protocol to include the KDR mutation. According to him, the very reason for having an MTB is precisely to be flexible: "to blindly follow the treatment assignment rules would amount to renounce reasoning, to merely apply priority rules". The TAC mentions that the MTB did already update rules in the past, and they can do it again. MB1 agrees while pointing out that they still have to decide whether to opt for Drug X or Y. The TPI argues that Drug Y has lower toxicity but is also more difficult to monitor because of possible cardiac side-effects. The MB1 suggests carrying out a one-week poll of all clinicians and biologists involved in the trial (about 50 people, although only 10 were attending the MTB meeting) about their preference for Drug X or Y. Following the oneweek poll, the official minutes of the meeting acknowledge that the final decision does not conform to the protocol: against treatment assignment rules, the MTB has decided to 
prescribe drug $\mathrm{Y}$ and to modify the protocol by adding KDR mutations to the list of targetable alterations.

In this excerpt, the trial PI contrasts MTBs with automatic decision-making by computerbased algorithms. Critics of MTB-based trials, in contrast, denounce the 'subjectivity' of this kind of decision-making that will render trial results difficult to assess and compare. A major US trial, $\mathrm{NCl}-\mathrm{MATCH}$, has consequently implemented an automated IT system for treatment assignment (Goldberg 2017). Conversely, the PI in our excerpt favours a flexible approach based on the ongoing assessment of the unfolding of a trial, whereby MTB members can decide to treat initial rules as obsolete and modify the protocol accordingly. Many leading precision-oncology trialists share his position, as exemplified by the following statement by the PI of several US genomic-driven trials (personal communication, August 2017):

Classically, algorithms for treatment decisions are locked down at the beginning of a trial. That was fine in the old days when science moved slowly. It is no longer fine. Algorithms must be flexible and incorporate new knowledge as it appears. ... A colleague who is $\mathrm{PI}$ of a molecularly driven trial was having a problem because her trial was not flexible. MSI-H patients [i.e. those with high levels of a genomic marker known as microsatellite instability] were considered unmatched because, at the time the trial was created, it was not known that MSI-H matched patients to [a new form of] immunotherapy. As a result, the unmatched arm was turning out better than the matched arm. That is nonsensical. Once it is known that something is a match, that scientific knowledge needs to be incorporated into the trial. "Locking" the algorithm at the beginning, which used to be considered sacrosanct, is no longer reasonable in the age of rapid scientific breakthroughs.

Indeed, as part of our fieldwork, we attended several oncology conferences during which speakers referred to the rapid obsolescence of knowledge about genomic variants and the need for more flexible or adaptive trials designs as a sine qua non for the development of precision oncology. This points to the temporally situated dimension of MTB activities, to which we now turn.

\subsection{The temporal dimension of MTBs}

In their analysis of the "age of genomic instability", Lappé and Landecker (2015: 161) define genomes as dynamic and heterogeneous between and within individuals. They accordingly centre their analysis on the "temporal axis" of genomic instability, arguing that "mutations that were previously thought to be stable, heritable and comparable between individuals are multiplying into a more complex landscape of molecular variants, many of which have 
unclear penetrance and are found in both 'normal' and diseased populations". They conclude (p. 179) that "it is not only the meaning of genomes and mutations that had shifted, but the very material objects of genomics have come to be understood as an unstable entity in time and space".

While the ethnographic scale of our analysis differs from that of Lappé and Landecker, we concur with their argument. Some of the excerpts included in the previous sections do indeed point to the temporal dimension of genomic data interpretation as enacted by MTBs. Knowledge about functional aspects of variants and expertise concerning their actionability is still in the making and rapidly evolving. We saw how MTBs can decide to break rules, and this kind of rule-breaking is often justified on the basis of temporal considerations, such as, for instance, new information on concurrent trials garnered at recent oncology meetings. The following excerpt illustrates this kind of versatile dynamic:

The patient has both a PI3Kinase mutation ad a KRAS amplification. According to trial rules, mutations have priority over amplicons. As stressed, however, by a biologist, this particular amplicon is "very beautiful", i.e. extremely high and thin. The oncologist PI argues that they should disregard the rules and target the amplicon because recent clinical trials have shown that drug X, which is available as part of this trial, works very well with altered KRAS: "Here, in the present case, there are clinical studies showing that when KRAS ... well, it's especially the case with mutations but also with amplifications, but when KRAS is altered, then the drug works very well".

Researchers, in a seemingly unstoppable flow of publications, introduce new variants and reassess the functional description of known variants on the basis of new evidence from recent clinical studies. The introduction of new technologies, such as 'saturation editing' (GenomeWeb 2018) also allows for a more detailed exploration of variants, thus further contributing to the rapid turnover of genomic information. For sure, medical work has always been subject to revisions following the production of new results or the emergence of new technologies, but in the case of genomic medicine these transformations are occurring at an unprecedented rate.

\subsection{MTBs as situated, 'ad-hoc' activities}

In addition to the temporal dimension, MTB activities are liable to a number of contingent or contextual constraints. Some of these factors refer to the specific framework within which different kinds of MTBs operate. MTBs for clinical trials, for instance, have to take into account the specific list of experimental or off-label drugs included in the trial protocol, and 
therefore also the molecular alterations that should be investigated, although, as we saw, MTB discussions can lead to protocol amendments. Routine clinical MTBs have, at least in principle, a broader manoeuvring range because oncologists can select drugs from all those available on the market. There are also intermediate situations: for instance, in the case of international trials, drugs available in one country are not necessarily available in another one, leading to MTB discussions about acceptable substitutes. Some MTBs are attached to 'treatment trials' that involve the prescription of drugs to patients, while others are part of 'triage trials' that centre on the genomic analysis of patients who are subsequently directed to available treatment trials. In short, there is a number of possible MTB configurations that constrain (but do not determine) the content of MTB activities, their interpretative work, and the actionability of molecular alterations found in patients. We refer to these contextual factors as the situatedness or indexicality of MTB activities (Suchman 2007).

At a different level, other contingent factors include the clinical peculiarities of patients, their therapeutic trajectories and co-morbidities as assessed by attending clinicians. Maybe less obviously, the presence or absence of particular MTB members (e.g., particularly outspoken or directive ones) also affect the course of MTB discussions. These factors point to the 'adhocness' of MTB activities, as exemplified by some of the excerpts we discussed in the previous section. As argued by Lynch (2013: 60) paraphrasing Suchman, "even the most elaborate plans and instructions for using novel technologies require users to deploy ad hoc procedures for getting the task done". In the case of MTBs, 'ad-hocness' refers to decisions made for a specific patient on a specific occasion based on considerations that are unique to that occasion. Ad hoc procedures include particular interpretations of the indexical elements regulating MTB activities. The following longish but instructive excerpt provides one among many other possible examples of this aspect of MTB decision-making:

The oncologist PI [TPI] introduces the case of a patient who has a BRCA2 germline mutation but no loss of the second allele [as required by treatment allocation rules], wondering whether the patient should nonetheless be eligible for a drug called Olaparib. He reminds MTB members that in previous cases they had "somewhat dogmatically" answered in the negative. However, he now argues, "it's a bit strange to say that this is a patient with a germline mutation known to be predictive of [olaparib] treatment, and we have access to this treatment, but we do not give it to him ... it's a little ... here we approach the limits of these algorithms ... personally I would put him in the Olaparib arm".

One of the biologists [BIO 1] points out that there is another possibility for this patient, because he also has a PIK3R1 mutation that can be treated with [drug X, an anti-AKT]. Another biologist [BIO 2] concurs with TPI that they could prescribe Olaparib because of the BRCA2 mutation, but he hesitates because "on the inactivating side ... it's true that there is no loss ...". The TPI interrupts him and the following discussion ensues: 
TPI: What do we know about the loss of the second allele? Because the loss of the second allele may very well be a loss by methylation or something like that.

BIO 1: Methylation for BRCA2 normally no, ... it's rare, well, it's not often described.

TPI: I would treat him with Olaparib ... otherwise it will be a little weird to justify that a patient who is BRCA2-mutated is treated with an anti-AKT.

BIO 2: Well, especially if he did not have that option [in previous clinical trials]. Because BRCA2 came out at -0.53 in log ratio ... sorry, at $-0.34 \ldots$ it doesn't look like a loss [of the second allele].

BIO 1: It's not a deletion.

TPI: Well, there could be some intragenic deletion or something like that, that we did not see, because [the analysis] was made with [a less sensitive technology].

BIO 2: Yes exactly, exactly! ... that could be the case, yes, absolutely! It's a very good point.

TPI: That's good, and as far as I'm concerned, personally I would treat this patient with

Olaparib especially because, in addition, the poor man already ended up in the placebo arm [of a previous trial of Olaparib vs. placebo], it would be a bit mean to tell him that we opted for an anti-AKT.

TPI [to the treating oncologist]: Do you agree with that?

Treating oncologist: Completely, it's the logical thing to do.

Administrative coordinator: Okay, so this patient will be oriented towards Olaparib.

As argued by Lynch (2011: 837), "far from [being] random or senseless", or a "disorderly 'mess'" (as many social scientists tend to treat them), ad hoc practices "relate to stable and intelligible orders of activity". In the present case, they pertain to the task of inscribing patients in a clinical trajectory as redefined by rapidly evolving and often fuzzy genomic information, thus making the very work of MTBs qua MTBs possible. As in a previous excerpt where the oncologist argued for a flexible interpretation of treatment assignment rules, the present excerpt shows how the experimental dimension blends with considerations concerning the compassionate care of individual patients.

\section{Conclusion}

Tumour sequencing leads to the identification of tumour-driving events that need to be summarized, interpreted, and prioritized in the context of current literature, drug-gene interaction databases, relevant clinical trials and known clinical actionability from knowledgebases (Good et al. 2014). These events also need to be related to clinical data from the case history of individual patients (Van Allen et al. 2013), for their main purpose is not to produce scientific facts, but to lead to clinical interventions, although practitioners insist on the dual purpose of bio-clinical studies in genomic medicine: to treat patients and to learn about the biopathology of cancer. As noted by Ghazani et al. (2017: 787), "assigning 
clinical meaning to each somatic and germ-line variant, including the therapeutic, prognostic, and diagnostic implications for individual patients, poses considerable difficulties in light of the inconsistent state of genome biological annotation". Confronted with this task, molecular oncologists have developed MTBs as sui generis institutions for the production of 'informed' data interpretation, both in the context of clinical trials and within routine, albeit increasingly experimental clinical settings. In this respect, readers might wonder whether MTB activities concern patients or research subjects, but as illustrated by some of the passages discussed in this paper and argued in more detail in another paper (Cambrosio et al. 2018), the fading of the boundary between research and care is one of the main characteristics of precision oncology.

MTBs mobilize a diverse set of human actors on both sides of the traditional clinical/scientific divide as well as a heterogeneous set of evidential resources, ranging from databases and knowledgebases to clinical trial results, previous clinical experience, and basic knowledge about mutations and pathways. As we saw in the fieldwork excerpts quoted in this paper, MTB activities qualify as: (a) distributed, insofar as they involve the detailed analysis and interpretation of genomic results by all MTB members across pre-established specialty boundaries, while also resting on the mobilization of devices and resources that link MTB members to other, extended bio-clinical collectives; (b) emergent, insofar they cannot be reduced to individual skills or expertise, but are the outcome of these peculiar, heterogeneous assemblages; and (c) open-ended and evolving, because of their temporal dimension, and the situated and ad hoc nature of MTB deliberations. While several entities (diseases, drugs, mutations) are simultaneously mobilized as part of MTB activities, the description and functional interpretation of genomic variants - in short: their actionability - is clearly at the core of these activities.

Although MTB meetings invariably begin with a clinical case report, this is quickly followed by a summary of genomic analysis results, which then leads to the discussion of the actionability of those genomic variants. Discussions can be more or less detailed, insofar as they can either lead to the almost automatic prescription of a given drug closely related to a specific alteration, or, alternatively, to complex debates about which variant should be prioritized, the reliability of the drug-variant match, or even to questioning the relevance or robustness of a genomic result because of its borderline nature or the lack of alignment with other known factors. It is important to emphasize that, rather than 'mere' laboratory results that count as just one component of a diagnosis, these discussions centre on bio-clinical entities - genomic variants - that threaten to displace more traditional clinical approaches, such as those based on organ-of-origin definitions of cancer, as shown by the recent approval of histology- or tumour-agnostic' drugs (Goldberg 2018). This is why we suggest that the 
emergence of MTBs signals the emergence of a new kind of clinic, a clinic of variants. In spite of all this, it could be objected that the clinic of variants does not change the basic rules that guide the validation of new clinical knowledge, which can be traced back to the early $20^{\text {th }}$ century (Marks 1997). We disagree, not least because the new clinic is part of an ongoing, broader shift that involves a major rethinking of the basic characteristics of clinical trials (e.g., Sharpless and Doroshow 2019, Nelson et al. 2014, Keating and Cambrosio 2011).

Given the limited scalability of MTBs, the expansion of the clinic of variants from experimental settings to routine clinical care, already well under way, is likely to require a reconfiguration of their organization and functioning. Often evoked as a possible solution (albeit with unclear referents), 'virtual MTBs' might for instance consist of temporally and spatially distributed networks relying on a sophisticated IT infrastructure. In such a scenario, treating physicians would be able to routinely access a set of resources managed by dedicated decision support teams and encompassing patient cohort management software, knowledgebases, and data visualization and analysis software (e.g., Eubank et al. 2016, Johnson et al. 2015). In other words, the peculiar MTB configurations we investigated in this paper will probably transit to new models, but the issues and problems we discussed in this paper, in particular the search for actionable interpretations, will continue to pose significant challenges to the clinic of variants.

Acknowledgements. Research for this paper was made possible by grants from the French National Cancer Institute (INCa 2014-123) and the Canadian Institutes of Health Research (MOP-133687). We would like to thank all the clinicians and scientists who gave us access to their teleconferences and who agreed to be interviewed, in some cases repeatedly. In particular, we would like to express our gratitude to Unicancer, the French hospital federation specialized in cancer care, without whose help we could not have completed this paper. Clinical researchers from the SAFIR 02 clinical trial (supported by 'Fondation ARC') also deserve special mention: their activities provided us with much of the material used in this paper. For reasons of confidentiality, we cannot mention the name of the several other practitioners and clinical trials we investigated as part of our project.

\section{REFERENCES}

Baszanger, I. (2012) One more chemo or one too many? Defining the limits of treatment and innovation in medical oncology, Social Science \& Medicine, 75, 5, 864-872.

Berg, M. (1996) Practices of reading and writing. The constitutive role of the patient record in medical work, Sociology of Health and Illness, 18, 4, 499-524.

Bourgeault, I., Dingwall, R., and de Vries, R. (2013) The SAGE Handbook of Qualitative Methods in Health Research. Los Angeles: SAGE.

Cambrosio, A., Limoges, C. and Hoffman, E. (1992) Expertise as a network. A case study of the controversies over the environmental release of genetically engineered organisms. In 
Stehr, N. and Ericson R.V. (eds) The Culture and Power of Knowledge. Inquiries into Contemporary Societies. Berlin: De Gruyter. Pp. 341-361.

Cambrosio, A., Keating, P., Vignola-Gagné, E., Besle, S., and Bourret, P. (2018) Extending experimentation. Oncology's fading boundary between research and care, New Genetics \& Society, 37, 3, 207-226.

Cambrosio, A., Campbell, J., Vignola-Gagné, E., Keating, P., et al. (forthcoming) 'Overcoming the bottleneck'. Knowledge architectures for genomic data interpretation in oncology. In Leonelli, S. and Tempini, N. (eds) Varieties of Data Journeys: Data Processing and Movements Within and Across Practices. Cambridge (MA): MIT Press.

Castel, P., Tassy, L., Lurkin, A., Blay, J.-Y., et al. (2012) Multidisciplinarity and medical decision, impact for patients with cancer. Sociological assessment of two tumour committees' organization, Bulletin Du Cancer, 99, 4, E34-E42.

DeLanda, M. (2016) Assemblage Theory. Edinburgh: Edinburgh University Press.

Dodier, N. and Barbot, J. (2016) La force des dispositifs, Annales. Histoire, Sciences Sociales, 71, 2, 421-450.

Erdmann, J. (2015) All aboard. Will molecular tumour boards help cancer patients? Nature Medicine, 21, 7, 655-656.

Eubank, M.H., Hyman, D.M., Kanakamedala, AD., Gardos, S.M., et al. (2016) Automated eligibility screening and monitoring for genotype-driven precision oncology trials, Journal of the American Medical Informatics Association, 23, 4, 777-781.

Eyal, G. (2013) For a sociology of expertise. The social origins of the autism epidemic, American Journal of Sociology, 118, 4, 863-907.

Foucault, M. (1994). Dits et écrits, 1954-1988, vol. 3 (1976-1979). Paris: Gallimard.

Galison, P. (1997) Image and Logic. A Material Culture of Microphysics. Chicago: University of Chicago Press.

Garfinkel, H. (1984) Studies in Ethnomethodology. Cambridge: Polity Press.

GenomeWeb. (2018) Saturation editing of BRCA1 exons reveals functional, non-functional variants. https://www.genomeweb.com/cancer/saturation-editing-brca1-exons-revealsfunctional-non-functional-variants\#.W9HobFJoQXo

Ghazani, A.A., Oliver, N.M., St Pierre, J.P., Garofalo, A., et al. (2017) Assigning clinical meaning to somatic and germ-line whole-exome sequencing data in a prospective cancer precision medicine study, Genetics in Medicine, 19, 7, 787-795.

Goldberg, P. (2017) NCl-MATCH keeps enrolling. Targeted mutations are proving to be less common than estimated, The Cancer Letter, 43, 27, 3-6.

Goldberg, P. (2018) FDA approves second drug for a site-agnostic indication, The Cancer Letter, 44, 44, 4-8.

Good, B.M., Ainscough, B.J., McMichael, J.F., Su, A.I. \& Griffith, O.L. (2014) Organizing knowledge to enable personalization of medicine in cancer, Genome Biology, 15:438. 
Hoefflin, R., Geissler, A.L., Fritsch, R., Claus, R., et al. (2018) Personalized clinical decision making through implementation of a molecular tumor board. A German single-center experience, JCO Precision Oncology, 2, 1-16.

Horak, P., Klink, B., Heining, C., Gröschel, S., et al. (2017) Precision oncology based on omics data. The NCT Heidelberg experience, International Journal of Cancer, 141, 5, 877-886.

Johnson, A., Zeng, J., Bailey, A.M., Holla, V., et al. (2015) The right drugs at the right time for the right patient. The MD Anderson precision oncology decision support platform, Drug Discovery Today, 20, 12, 1433-1438.

Keating, P. and Cambrosio, A. (2011) Clinical trials in the age of personalized medicine, Journal of Medicine and the Person, 9, 91-98.

Knepper, T.C., Bell, G.C Hicks, J.K., Padron, E., et al. (2017) Key lessons learned from Moffitt's molecular tumor board. The clinical genomics action committee experience, The Oncologist, 22, 2, 144-151.

Kuroki, L., Stuckey, A., Hirway, P., Raker, C. A., et al. (2010) Addressing clinical trials. Can the multidisciplinary tumor board improve participation? A study from an academic women's cancer program, Gynecologic Oncology, 116, 3, 295-300.

Lappé, M. and Landecker, H. (2015) How the genome got a life span, New Genetics \& Society, 34, 2, 152-176.

Lynch, M. (2011) Ad hoc special section on ethnomethodological studies of science, mathematics, and technical activity. Introduction, Social Studies of Science, 41, 6, 835837.

Lynch, M. (2013) At the margins of tacit knowledge, Philosophia Scientiæ, 17, 3, 55-73.

Marks, H.M. (1997) The Progress of Experiment: Science and Therapeutic Reform in the United States, 1900-1990. Cambridge: Cambridge University Press.

Mateo, J., Chakravarty, D., Dienstmann, R., Jezdic, S., et al. 2018. A framework to rank genomic alterations as targets for cancer precision medicine. The ESMO Scale for Clinical Actionability of Molecular Targets (ESCAT), Annals of Oncology, 168, 4, 584-588.

McGowan, M.L., Ponsaran, R.S., Silverman, P., Harris, L.N., and Marshall, P.A. (2016) ‘A rising tide lifts all boats'. Establishing a multidisciplinary genomic tumor board for breast cancer patients with advanced disease, BMC Medical Genomics, 9:71.

McGraw, S.A., Garber, J., Jänne, P.A., Lindeman, N., et al. (2017) The fuzzy world of precision medicine. Deliberations of a precision medicine tumor board, Personalized Medicine, 14, 1, 37-50.

Nelson, N., Keating, P. and Cambrosio, A. (2013) On being 'actionable'. Clinical sequencing and the emerging contours of a regime of genomic medicine in oncology, New Genetics \& Society, 32, 4, 405-428. 
Nelson, N., Keating, P., Cambrosio, A., Aguilar-Mahecha, A. and Basik, M. (2014) Testing devices or experimental systems? Cancer clinical trials take the genomic turn, Social Science \& Medicine, 111, 74-83.

Rabeharisoa, V. and Bourret, P. (2009) Staging and weighting evidence in biomedicine. Comparing clinical practices in cancer genetics and psychiatric genetics, Social Studies of Science, 39, 5, 691-715.

Schwaederle, M., Parker, B.A., Schwab, R.B., Fanta, P.T., et al. (2014) Molecular tumor board. The University of California-San Diego Moores Cancer Center experience, The Oncologist, $19,6,631-636$.

Sharpless, N.E., and Doroshow, J.H. (2019) Modernizing clinical trials for patients with cancer, JAMA, 321: 447-448.

Stoeklé, H.C., Mamzer-Bruneel, M.F., Frouart, C.H., Le Tourneau, C., et al. (2017) Molecular tumor boards: Ethical issues in the new era of data medicine, Science and Engineering Ethics, 24, 1, 307-322.

Suchman, L.A. (2007) Human Machine Configurations: Plans and Situated Actions. Cambridge: Cambridge University Press.

Tafe, L.J., Gorlov, I.P., de Abreu, F.B., Lefferts, J.A., et al. (2015) Implementation of a molecular tumor board. The impact on treatment decisions for 35 patients evaluated at Dartmouth-Hitchcock Medical Center, The Oncologist, 20, 9, 1011-1018.

Timmermans, S. and Tavory, I. (2012) Theory construction in qualitative Research. From grounded theory to abductive analysis, Sociological Theory, 30, 3, 167-186.

Timmermans, S., Tietbohl, C., and Skaperdas, E. (2017) Narrating uncertainty. Variants of uncertain significance (VUS) in clinical exome sequencing, BioSocieties, 12, 3, 439-458.

Van Allen, E.M., Wagle, N. \& Levy, M.A. (2013) Clinical analysis and interpretation of cancer genome data, Journal of Clinical Oncology, 31, 15, 1825-1833.

Vignola-Gagné, E., Keating, P. and Cambrosio, A. (2017) Informing materials. Drugs as tools for exploring cancer mechanisms and pathways, History and Philosophy of the Life Sciences, 39:10.

Wagner, A.H., Walsh, B., Mayfield, G., Tamborero, D., et al. (2018) A harmonized metaknowledgebase of clinical interpretations of cancer genomic variants, bioRxiv, http://dx.doi.org/10.1101/366856. 